\section{Response to Knoppers et al.}

To the Editor: We appreciate the opportunity to respond to the comments of Knoppers and colleagues ${ }^{1}$ regarding the American College of Medical Genetics and Genomics (ACMG) statement, "Patient re-contact after revision of genomic test results: points to consider," ${ }^{2}$ recently e-published before print in Genetics in Medicine. The comments in Knoppers' letter, however, reflect a misunderstanding of our document and conflate the clinical and research settings. We reiterate that the ACMG statement reflects practice of American clinical geneticists who provide direct patient services. It does not speak to the research setting.

Current American clinical and legal standards and fundamental ethical principles inform our Points to Consider. We clearly state that reasonable efforts should be made by providers (not necessarily only physicians) to contact the patient (Points to Consider \#8). These efforts may encounter "resource constraints" that may be similar or dissimilar to those in the research setting. The letter of Knoppers et al. points out that the American Society of Human Genetics draft position ${ }^{3}$ limits researchers' "duty to re-contact" to the duration of the project funding. On the other hand, clinicians' concerns that patients be properly informed of revised interpretation may extend indefinitely after the original consultation. Given the practicalities of contacting patients potentially years after the results of a genetic test were first reported, it is neither reasonable nor realistic to promise more than providers can deliver. Despite best efforts on the part of laboratories and clinicians, re-contact cannot be guaranteed unless the patient initiates the process.

The ACMG statement does not place sole responsibility for re-contact on anyone; rather the statement stresses a collaborative approach. As highlighted in our Points to Consider, the ACMG recognizes that its position may well evolve with developments in molecular technology and variant interpretation, electronic record and communication technology, and the legal environment. Therefore, the ACMG
Points to Consider is meant as the best approach on these difficult issues in the current context of clinical care.

\section{DISCLOSURE}

The authors declare no conflicts of interest.

Publisher's note: Springer Nature remains neutral with regard to jurisdictional claims in published maps and institutional affiliations.

Karen L. David, MD, MS (10) ${ }^{1}$, Robert G. Best, $P h D^{2}$, Leslie Manace Brenman, MD, MPhil ${ }^{3}$, Lynn Bush, PhD, $M S^{4,5}$, Joshua Deignan, $P h D^{6}$, David Flannery, $M D^{7}$,

Jodi D. Hoffman, $M D^{8}$, Ingrid Holm, $M D, M P H^{9}$,

David T. Miller, MD, PhD ${ }^{4}$, James O'Leary, MBA and

Reed E. Pyeritz, $M D, P h D^{10}$

${ }^{1}$ Division of Genetics, Department of Medicine, New York-Presbyterian Brooklyn Methodist Hospital, Brooklyn, NY, USA; ${ }^{2}$ University of South Carolina School of Medicine Greenville and Greenville/Prisma Health System, Greenville, SC, USA; ${ }^{3}$ Regional Precision Tracking and Genetics Department, Kaiser Permanente Northern California, Oakland, CA, USA; ${ }^{4}$ Division of Genetics and Genomics, Boston Children's Hospital, Boston, MA, USA; ${ }^{5}$ Pediatric Clinical Genetics, Columbia University Medical Center, New York, NY, USA; ${ }^{6}$ Department of Pathology and Laboratory Medicine, David Geffen School of Medicine, University of California Los Angeles, Los Angeles, CA, USA; ${ }^{7}$ Center for Personalized Genetic Healthcare, Genomic Medicine Institute, Cleveland

Clinic, Cleveland, OH, USA; ${ }^{8}$ Division of Genetics, Boston Medical Center, Boston, MA, USA; ${ }^{9}$ Division of Genetics and Genomics and the Manton Center for Orphan Disease Research, Boston Children's Hospital and Department of Pediatrics, Harvard Medical

School, Boston, MA, USA; ${ }^{10}$ Division of Translational Medicine and Human Genetics, University of Pennsylvania School of Medicine, Philadelphia, PA, USA. Correspondence: Karen L. David (kld9001@nyp.org)

\section{REFERENCES}

1. Knoppers BM, et al. Letter: Relearning the 3 R's: reinterpretation, Recontact, and Return of Genetic Variants

2. David KL, Best RG, Brenman LM, ACMG Social Ethical Legal Issues Committee, et al. Patient re-contact after revision of genomic test results: points to consider-a statement of the American College of Medical Genetics and Genomics (ACMG). Genet Med. 22 Dec 2018; https://doi. org/10.1038/s41436-018-0391-z [Epub ahead of print].

3. Levy HP. Duty to re-contact in the research environment; the ASHG draft position statement presented ASHG Annual Meeting, San Diego, October 19, 2018.

Advance online publication 11 April 2019. doi:10.1038/s41436-019-0496-Z 